\title{
EVOLUCIÓN PALEOAMBIENTAL DURANTE EL PLIOCENO INFERIOR EN LA CUENCA DE ALMERÍA- NÍJAR (SE DE ESPAÑA) SEGÚN LAS ASOCIACIONES DE FORAMINÍFEROS BENTÓNICOS
}

\author{
Ana Belén PÉREZ-MUÑOZ, Rocío MÁRQUEZ-CRESPO, \\ Jesús YESARES-GARCÍA, Isabel María SÁNCHEZ-ALMAZO \\ y Julio AGUIRRE
}

Dpto. de Estratigrafía y Paleontología, Facultad de Ciencias, Fuentenueva, s/n. Universidad de Granada. 18071 Granada. (Direcciones electrónicas de contacto: sanchez@goliat.ugr.es y jaguirre@goliat.ugr.es )

Pérez-Muñoz, A.B., Márquez-Crespo, R., Yesares-García, J., Sánchez-Almazo, I.M. y Aguirre, J. 2001. Evolución paleoambiental durante el Plioceno inferior en la cuenca de Almería-Níjar (SE de España) según las asociaciones de foraminíferos bentónicos. [Palaeonvironmental evolution during the early Pliocene of the Almería-Níjar basin (SE Spain) based on benthic foraminifer assemblages.] Revista Española de Paleontologia. $n^{0}$ extraordinario, 21-34. ISSN 0213-6937.

\begin{abstract}
Changes in benthic foraminifer assemblages in relation to palaeoenvironmental variations have been studied in the lower Pliocene deposits of Palmo de Salas, $N$ of Almería-Níjar basin (SE Spain). Changes in foraminifer assemblages indicate an upward shallowing trend, from outer shelf deposits at the bottom of the section to inner platform facies at the top. Binary cluster analysis reveals the succession of three groups that correlate with this shallowing trend. The foraminifer assemblage at the base of the section is dominated by Cassidulina neocarinata $(\approx 50 \%)$ that characterises the deepest deposits, formed in dysaerobic conditions. The shallowest facies are dominated by Ammonia, Nonion, Cribroelphidium and Elphidium. The middle platform settings are represented by diverse and abundant benthic foraminifer assemblages, which are dominated by Nonion asterizans, Elphidiumn spp., Lenticulina calcar, Cibicides spp. and Asterigerinata planorbis. Two minor upward shallowing cycles can be recognised: the lower cycle corresponds to the lower half part of the section, and the upper one to the upper half part. The boundary between the two cycles is inferred by a change in the depth distribution of foraminifer species, in the foraminiferal assemblages and in the planktonic/benthic ratio $(P / P+B)$. Finally, a quantitative $Q$ mode cluster analysis allows to recognise benthic foraminifer groups controlled by palaeoenvironmental factors other than bathimetry. Thus, it separates one group, dominated by Bulimina. Cassidulina, Hopkinsina and Brizalina, that characterises dysaerobic conditions. Q-mode cluster analysis also differentiates a benthic foraminifer assemblage dominated by typically epiphitic species, such as Elphidium crispum, E. macellum, Cribroelphidium decipiens, Cibicides refulgens and C. lobatulus.
\end{abstract}

Keywords: Benthic foraminifera, palaeoecology, palaeoenvironmental evolution, early Pliocene, AlmeríaNíjar basin, SE Spain.

\section{RESUMEN}

En este trabajo se estudian las variaciones observadas en las asociaciones de foraminíferos bentónicos en relación con cambios paleoambientales, en los sedimentos del Plioceno inferior del Palmo de Salas, $\mathrm{N}$ de la cuenca de Almería-Níjar (SE de la Península Ibérica). Los cambios en las asociaciones de foraminíferos bentónicos ponen de manifiesto una somerización hacia el techo, desde facies de plataforma externa en la base de la sección, a facies de plataforma interna somera en el techo. Un análisis "cluster" binario cualitativo pone de manifiesto un relevo de tres agrupaciones diferentes que refleja dicha somerización. La base de la sección está caracterizada por una asociación de foraminíferos bentónicos dominada en casi un 50\% por Cassidulina neocarinata. Estos 
materiales corresponden a los depósitos más profundos, formados en un medio disaeróbico. Las facies más someras, hacia el techo de la sección, están caracterizadas por una asociación dominada por los géneros Ammonia, Nonion, Cribroelphidium y Elphidium. Las facies de plataforma media están caracterizadas por asociaciones más diversas y con gran abundancia de especies, dominando Nonion asterizan.s, Elphidium spp., Lenticulina calcar, Cibicides spp. y Asterigerinata planorbis. Dentro de esta secuencia general somerizante se pueden diferenciar dos ciclos de somerización menores. La base del segundo ciclo queda reflejada, además de por un cambio en las asociaciones, por un incremento substancial en la diversidad y en la relación planctónicos/bentónicos $(\mathrm{P} / \mathrm{P}+\mathrm{B})$. Otros máximos de diversidad detectados en la sección pueden también corresponderse con entradas de nutrientes a la cuenca. Finalmente, un análisis "cluster" cuantitativo en modo "Q" pone de manifiesto, además de las asociaciones que están controladas por la batimetría, la existencia de subgrupos que caracterizan ambientes empobrecidos en oxígeno (disaeróbicos), dominados por los géneros Bulimina, Hopkinsina, Cassidulina y Brizalina, y ambientes con un fondo cubierto por algas, caracterizados por una asociación de foraminíferos típicamente epifíticos (Elphidium crispum, E. macellum, Cribroelphidium decipiens, Cibicides refulgens y C. lobatulus).

\section{Palabras clave: Foraminíferos bentónicos, paleoecología, evolución paleoambiental, Plioceno inferior, cuenca de Almería-Níjar, SE de España.}

\section{INTRODUCCIÓN}

Desde finales del siglo pasado se han realizado numerosos estudios en los que se analiza la distribución ecológica de los foraminíferos bentónicos y su aplicación potencial al registro fósil, tomando siempre como referencia la distribución y los requerimientos ecológicos de las especies actuales o de los representantes equivalentes de especies extintas. La gran mayoría de estos estudios (paleo)ecológicos han intentado establecer modelos de zonación batimétrica de diferentes asociaciones de foraminíferos bentónicos (ver recopilaciones en Douglas, 1979; Sen Gupta, 1980; Miller, 1980; Van Morkhoven et al., 1986; Murray, 1991). No obstante, estos modelos son muy generales y de validez limitada, ya que las diferentes especies de foraminíferos bentónicos muestran rangos batimétricos muy amplios (100-200 m, e incluso mayores) en las diferentes regiones marinas. Se ha podido reconocer, así, que las asociaciones características de plataforma interna están fundamentalmente dominadas por los géneros Asterigerinata, Ammonia, Elphidium, Amphistegina, Astrononium y Cancris, mientras que Bulimina, Bolivina, Cibicides, Eponides y Fursenkoina, entre otros géneros, son más abundantes en ambientes de plataforma externa y talud superior. Hacia regiones más profundas, se produce una disminución en importancia de algunos de los géneros anteriores y toman el relevo los géneros Cassidulina, Cibicidoides, Melonis, Uvigerina, Pullenia y Gyroidinoides, junto con una mayor proporción de foraminíferos bentónicos aglutinantes (por ejemplo, Parker, 1958; Berger, 1979; Murray, 1991; Gooday, 1994; De Rijk et al., 1999).

Estos patrones de distribución de asociaciones con la profundidad, en ocasiones, se ven substancialmente modificados, ya que hay otra serie de factores físicoquímicos y biológicos que ejercen un mayor control sobre la distribución de estos organismos. Entre estos factores cabe destacar: tipo de substrato, hábito alimenticio, patrones de circulación, disponibilidad de alimentos, contenido en oxígeno disuelto en las aguas de fondo y/o en la columna de sedimento, contenido en materia orgánica y nutrientes y salinidad (Sen Gupta, 1980; Jorissen, 1987; Murray, 1991; De Rijk y Troelstra, 1997).

Todos estos factores actúan conjuntamente sobre especies y asociaciones de especies de foraminíferos bentónicos. Por tanto, el estudio de la distribución de dichas asociaciones aporta una información muy precisa sobre la evolución ambiental de una cuenca.

La escasa disponibilidad de alimento, además, condiciona la existencia de asociaciones dominadas por formas predominantemente oligotróficas, tales como Cibicides y Melonis (Kahio, 1994; Schmiedl et al., 1997). Por el contrario, en áreas eutróficas, normalmente relacionadas con afloramientos de aguas profundas ("upwelling") o con la influencia de aportes continentales, se dispara la abundancia de especies de foraminíferos bentónicos mientras que la diversidad suele disminuir (por ejemplo, Murray, 1995; Loubere y Fariduddin, 1999). El continuo flujo de partículas orgánicas hacia el fondo y/o el aumento de la estratificación de las aguas provoca una disminución del contenido en oxígeno disuelto en el fondo. Este proceso produce una somerización del nivel de potencial redox dentro de la columna de sedimento, acercándose hacia la interfase agua-sedimento. El mantenimiento prolongado de estas condiciones puede generar fondos empobrecidos en oxígeno (disaeróbicos) e, incluso, anóxicos. Estas condiciones extremas favorecen el desarrollo de comunidades poco diversas dominadas por especies oportunistas y con gran tolerancia a variaciones en el contenido en oxígeno, como son, por ejemplo, los bolivinítidos (Katz y Thunell, 1984; Moodley y Hess, 1992; Murray, 1991, 1995; Sen Gupta y Machain-Castillo, 1993; Kaiho, 1994; Schmiedl et al., 1997). En estas condiciones, el substrato, al igual que las corrientes de fondo, pueden jugar un papel determinante en la distribución de las asociaciones de foraminíferos bentónicos (Langer, 1993; Kitazato, 1994; MacKensen et al., 1995; Schmiedl et cl., 1997; Alve y Murray, 1999).

El hecho de estudiar el efecto conjunto de los diferentes factores ecológicos sobre las poblaciones de foraminíferos bentónicos ha despertado un interés especial 


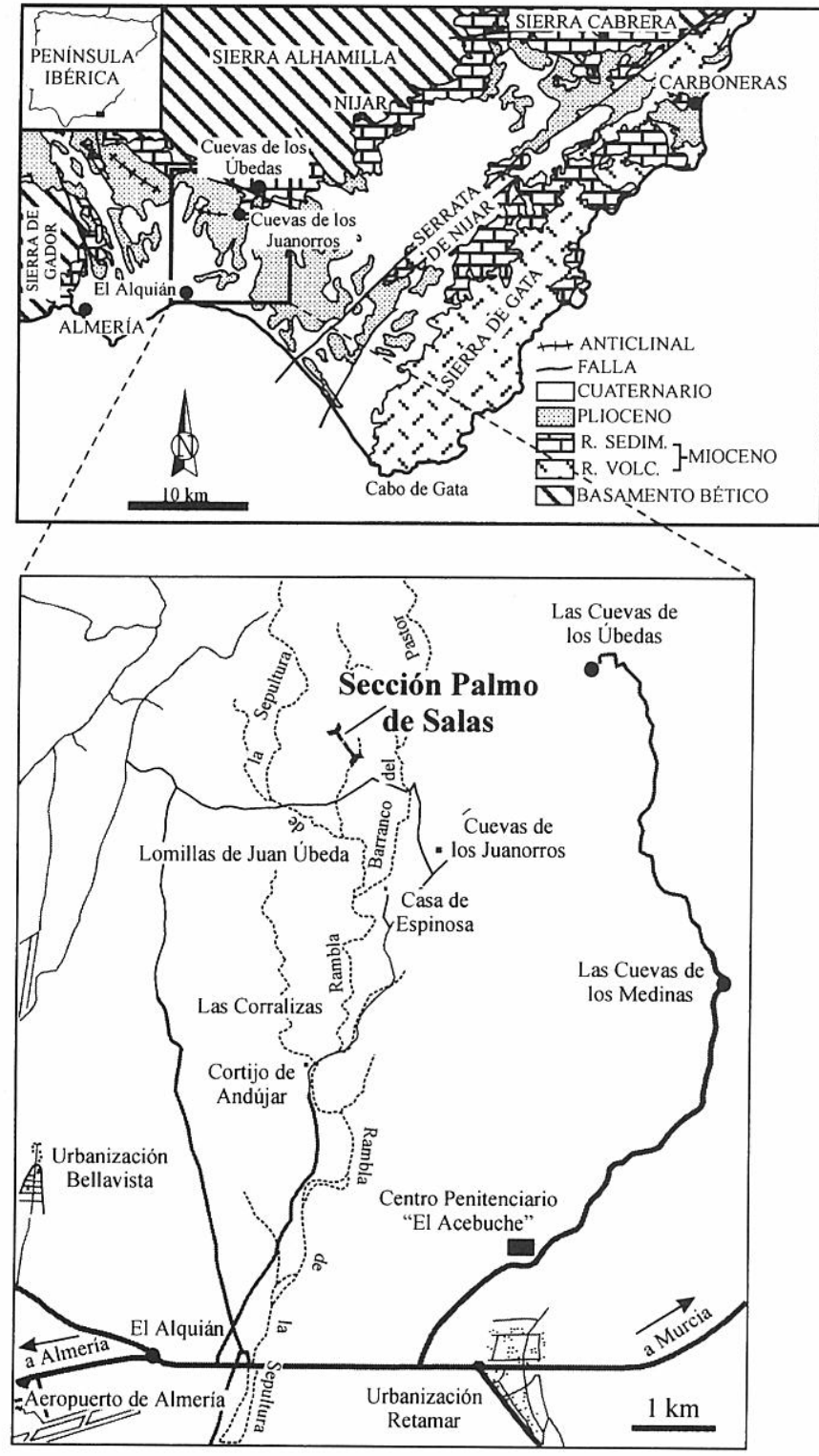

Figura 1. Mapa geológico de las cuencas de Almería-Níjar y de Carboneras, situadas al sur de la alineación montañosa constituida por Sierra Alhamilla y Sierra Cabrera. Como puede observarse, la sección estudiada en este trabajo, sección Palmo de Salas, se encuentra situada en el flanco $\mathrm{N}$ de un anticlinal localizado en el margen $\mathrm{N}$ de la cuenca de Almería-Níjar.

Geological map of the Almería-Nijar and Carboneras basins, located south of Sierra Alhamilla and Sierra Cabrera. The studied section, Palmo de Salas section, is in the northern part of an anticline located in the north of the Almeria-Nijar basin.

en la última década, ya que ha potenciado el uso de estos organismos en campos tales como la oceanografía. En este sentido, en este trabajo se analizan las asociaciones de foraminíferos bentónicos en los sedimentos del Plioceno inferior de la cuenca de Almería-Níjar (SE de España) para establecer un modelo paleoambiental y de evolución secuencial durante el depósito de estos materiales. Los resultados obtenidos ponen de manifiesto la existencia de cambios paleoambientales que hasta ahora no habían sido detectados estudiando exclusivamente los sedimentos y/o las asociaciones de macroinvertebrados (Aguirre et al., 1996; Aguirre, 1995, 1996, 1998).

\section{LOCALIZACIÓN Y DESCRIPCIÓN DE LA SECCIÓN ESTUDIADA}

La sección estudiada se localiza en el Palmo de Salas, en el margen $\mathrm{N}$ de la cuenca de Almería-Níjar, aproximadamente $7 \mathrm{~km}$ al $\mathrm{N}$ de El Alquián y cerca de las Cuevas de los Juanorros (Fig. 1).

El relleno neógeno de la cuenca comienza con margas de edad Mioceno medio (Serrano, 1990; Montenat et al., 1990) y finaliza con depósitos mixtos terrígeno-carbonatados del Plioceno superior (Aguirre y Jiménez, 1997, 1998; Aguirre, 1998; Aguirre y Sánchez-Almazo, 1998). Por encima de los materiales neógenos se disponen discordantemente depósitos de playa y de abanicos aluviales atribuidos al Cuaternario (Goy y Zazo, 1982).

Dentro del conjunto Plioceno de la cuenca de AlmeríaNíjar se diferencian dos unidades (denominadas Unidades I y II) separadas por una discordancia. La Unidad I, objeto de estudio en este trabajo, es de edad Plioceno inferior-base del Plioceno superior (Aguirre, 1998). Los sedimentos pertenecientes a esta unidad se depositaron en un amplio golfo abierto hacia el Mediterráneo, cuyos límites eran los relieves béticos de las sierras de Gádor, Alhamilla y Cabrera y los relieves volcánicos miocénicos de la Serrata de NíjarSierra de Gata (Aguirre, 1998). Diversos sistemas deltaicos aportaron grandes volúmenes de sedimentos terrígenos a la cuenca (Aguirre, 1998), siendo el más importante el delta Abrioja, localizado en el extremo NO de la cuenca (Postma, 1983).

En la sección Palmo de Salas se reconocen las dos unidades pliocenas antes citadas. La Unidad I, que alcanza un espesor máximo de unos $70 \mathrm{~m}$, buza unos $25^{\circ}$ al NNE. La base de la unidad no aflora en este punto, aunque sí lo hace más al $\mathrm{N}$ y al $\mathrm{E}$, donde se dispone discordantemente sobre margas y yesos de edad Messiniense. En la base de la sección estudiada afloran margas gris-azuladas, localmente muy fosilíferas y con algunos cantos muy dispersos (Fig. 2).

Por encima se pasa gradualmente a limos verdosoamarillentos, arenas finas, arenas medias-gruesas muy micáceas y, finalmente, a conglomerados (Fig. 2). En los limos y arenas finas se intercalan capas de concentraciones de bioclastos de 7 a $10 \mathrm{~cm}$ de espesor máximo con bases irregulares. Dichas acumulaciones se interpretan como tempestitas distales. Estos materiales corresponden a las facies de prodelta del delta Abrioja. En las arenas mediasgruesas y conglomerados aparecen paquetes de calcarenitas y calcirruditas de hasta $1 \mathrm{~m}$ de potencia, con muros erosivos y superficies de amalgamación internas que se interpretan como tempestitas proximales (Aguirre, 1996; Aguirre et al., 1996). El conjunto de estos materiales corresponde a las facies de frente deltaico. 
La sección finaliza con un nivel de acumulación de nódulos de algas coralináceas (rodolitos) incluidos en una matriz bioclástica con algunos cantos de cuarcita, que se superpone a las arenas y conglomerados. Son materiales formados en un ambiente de plataforma somera afectada por el oleaje de tormentas fuera del alcance directo del material siliciclástico.

\section{MATERIAL Y METODOLOGÍA}

El muestreo se ha realizado a intervalos regulares de $5 \mathrm{~m}$. Se recogieron un total de 15 muestras (Fig. 2) de alrededor de $1 \mathrm{~kg}$ de peso en seco, de las cuales se tamizaron aproximadamente $100 \mathrm{gr}$. Las muestras fueron procesadas en el laboratorio mediante la técnica de levigado habitual, utilizando para ello tamices de luz de malla de $1 \mathrm{~mm}$ y 0,125 mm. Posteriormente, se realizó un análisis semicuantitativo siguiendo el protocolo propuesto por Murray (1991). Así, en cada muestra se contaron 300 individuos de foraminíferos al azar para obtener la proporción entre foraminíferos planctónicos y bentónicos (relación $\mathrm{P} / \mathrm{P}+\mathrm{B}$ ). De igual forma, se contaron al azar 300 individuos de foraminíferos bentónicos en cada una de las muestras para obtener la abundancia de especies y la diversidad.

La diversidad, entendida como el número de especies y el reparto de cada una de ellas en las muestras, se ha expresado con los siguientes índices: 1) número total de especies en cada muestra; 2) heterogeneidad, H, o índice de diversidad de Shannon-Wiener (=Shannon-Weaver); y, 3) índice de equitatividad, E. La similitud entre las curvas definidas por estos parámetros a lo largo de la sección ha sido evaluada mediante un análisis no paramétrico de correlación de Spearman.

Para caracterizar las asociaciones de especies de foraminíferos bentónicos se han analizado, por un lado, las especies más abundantes en las muestras, y, por otro, se ha realizado un análisis multivariante. En el primer caso, se han considerado aquellas especies que están representadas por más del $5 \%$ de la asociación, obteniéndose, así, la distribución de abundancia de especies. En el segundo caso, se han realizado dos tipos de análisis "cluster", uno binario y otro cuantitativo en modo "Q" (agrupación entre muestras). El primero evalúa de forma cualitativa la similitud entre pares de muestras, considerando exclusivamente presencia $(=1)$ y ausencia $(=0)$ de especies (Davis, 1986; Magurran, 1989; Swan y Sandilands, 1995). Existen numerosos índices de similitud, aunque los más usados son los índices de Jaccard y de Sorenson (Magurran, 1989). En este trabajo se ha utilizado el índice de Sorenson.

Por su parte, el análisis "cluster" cuantitativo tiene en cuenta la abundancia relativa de las diferentes especies y los resultados obtenidos se complementan con los del análisis anterior. El "cluster" se ha obtenido a partir del método del encadenamiento medio entre grupos ("average linkage") (Shi, 1993) usando como distancia de similitud entre muestras el índice de correlación de Pearson.

El uso de los dos tipos de análisis permite hacer interpretaciones complementarias de las agrupaciones resultantes de ambos procedimientos. En los dos tipos de análisis se han incluido todas las especies identificadas; un total de 103 taxones reconocidos en 14 muestras (la muestra XI se ha excluido de los análisis por la ausencia de foraminíferos).

\section{ASPECTOS TAFONÓMICOS}

En relación con los aspectos tafonómicos es de resaltar el buen grado de conservación que presentan las conchas, en especial la muestra I. En esta muestra las conchas de foraminíferos se encuentran prácticamente sin ningún tipo de alteración tafonómica (desgaste, fragmentación, disolución, etc.). En cuanto al resto de las muestras la preservación es variable, aunque dominan los restos bien conservados. Se constata la presencia de conchas que muestran un cierto grado de desgaste y recristalización, especialmente hacia el techo de la sección, lo que impide, en determinadas ocasiones, su identificación a nivel específico. No obstante, no existen evidencias de conservación diferencial de especies que sesguen los parámetros de las comunidades originales. Todo esto sugiere que no hay una mezcla sustancial entre organismos que se desarrollaron en ambientes muy diferentes (Brett, 1995), generándose asociaciones autóctonas-paraautóctonas que permiten hacer inferencias de las condiciones paleoambientales donde se formaron.

Es de destacar, no obstante, que Elphidium crispum (Linneo) y diversas especies del género Lenticulina suelen encontrarse fragmentadas, afectadas por fenómenos de "corrasión" (sensu Brett y Baird, 1986) y por disolución parcial debido, probablemente, al efecto de organismos perforadores. En el caso particular de las conchas de Lenticulina spp. se observa un gradiente entre cinco tipos básicos de conservación (Pérez-Muñoz et al., 2000): 1) conchas perfectamente conservadas; 2) conchas con un surco a lo largo de toda la periferia (facetas periféricas); 3) conchas carenadas o no carenadas a las que les faltan los umbos en ambos laterales; 4) umbos aislados; y, 5) conchas con una única mitad espiral conservada. Esta conservación diferencial puede explicarse por un doble proceso: a) debido a una mezcla entre individuos que han sido retrabajados desde zonas más someras y otros que se acumularon aproximadamente en el lugar donde vivían; $y$, b) debido a un mayor tiempo de exposición sobre el fondo (Pérez-Muñoz et al., 2000).

\section{RESULTADOS}

\section{DIVERSIDAD}

Aunque existen algunas diferencias entre las curvas obtenidas con los diferentes índices usados, las tendencias observadas son similares (Fig. 2). Un análisis de correlación de Spearman confirma esta similitud (heterogeneidad / equitatividad $\rho=0,943 / \mathrm{p}=0,0007$; heterogeneidad $/ \mathrm{N}^{\circ}$ de especies $\rho=0,912 / \mathrm{p}=0,001$; equitatividad $/ \mathrm{N}^{\circ}$ de especies $\rho=0,763 / \mathrm{p}=0,006$. Donde $\rho$ es el índice de correlación de Spearman y p es la 

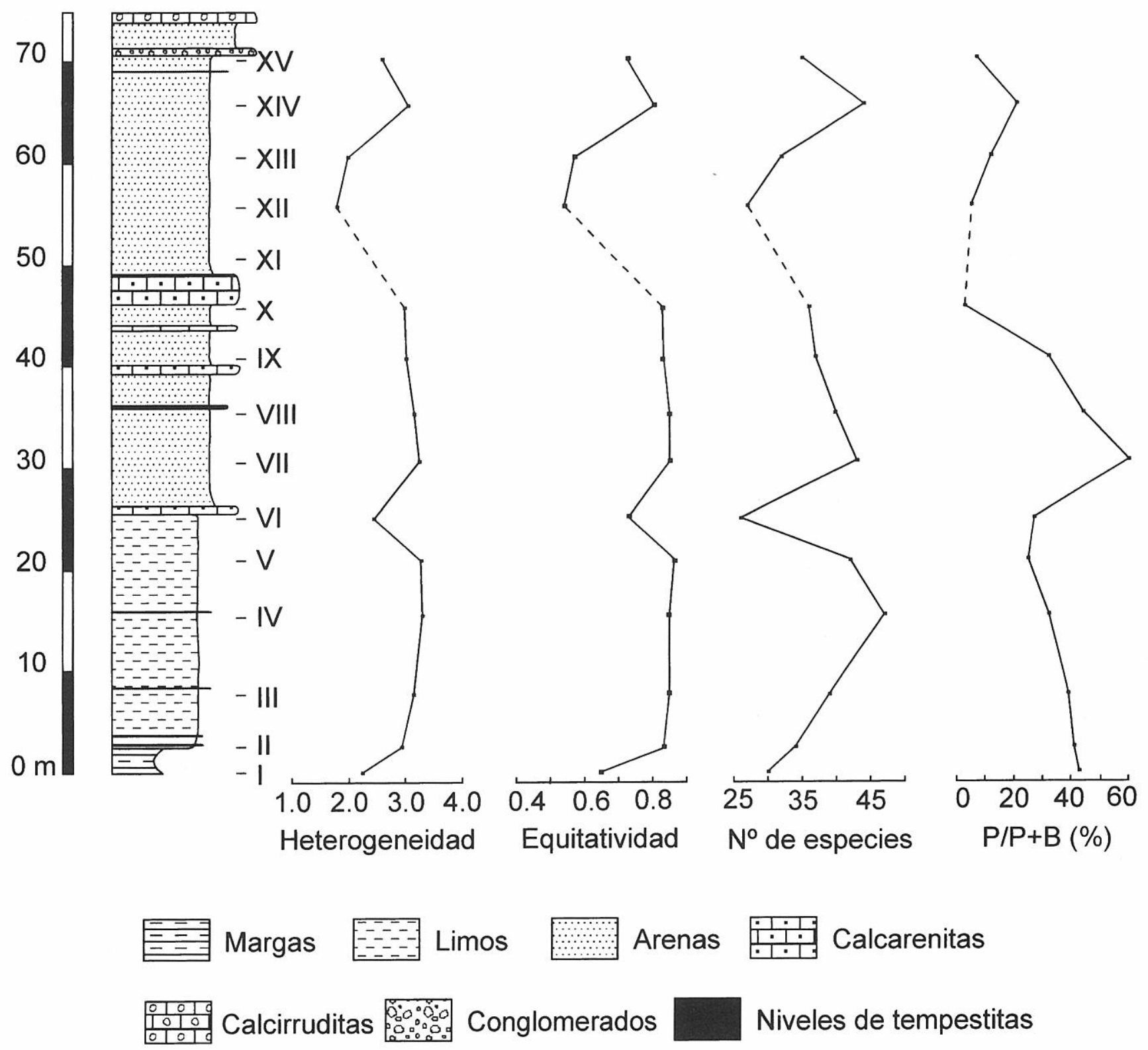

Figura 2. Columna estratigráfica de la sección Palmo de Salas donde se representa con números romanos del I al XV la posición de las muestras estudiadas. En la muestra XI no se ha identificado ningún resto de foraminífero, por lo que ha sido excluida de los análisis. Además, se muestran las curvas que representan el índice de diversidad de ShannonWiener (=Shannon-Weaver) o Heterogeneidad $(\mathrm{H})$, el índice de Equitatividad (E) y el número de especies. Obsérvese la existencia de máximos y mínimos en las tres curvas que, salvo por el pico de la muestra IV, coinciden en todas ellas. La última curva representa la relación entre foraminíferos planctónicos y bentónicos $(\mathrm{P} / \mathrm{P}+\mathrm{B})$. Este parámetro muestra una tendencia general a la disminución desde la base de la sección hacia techo, salvo por la existencia de dos máximos, uno en la muestra VII y otro en la muestra XIV.

Stratigraphic column of the Palmo de Salas section, indicating the position of the samples (I to XV). Sample XI has no foraminifer remains and, therefore, it has been excluded from statistical treatments. Curves of the ShannonWiener indice (=Shannon-Weaver) or heterogeneity $(H)$, evenness $(E)$ and number of species are included. There are several maxima and minima which coincide in all the curves, except for the peack of species number in sample $I V$. The last curve represents the planktonic-benthic ratio $(P / P+B)$. This curve shows a decreasing trend along the section, except for two maxima in samples VII and XIV.

probabilidad). Por tanto, en lo sucesivo, se referirá a diversidad en términos generales para expresar cómo varía a lo largo de la sección el número de especies, sin ninguna implicación según los diferentes parámetros usados, a excepción de los casos en que se especifica.
En general, la diversidad muestra un patrón de máximos y mínimos a lo largo de la sección. Desde la base de la sección se produce un incremento paulatino en el número de especies hasta alcanzar un máximo en la muestra IV. Esta tendencia, sin embargo, no se pone de 


\begin{tabular}{|c|c|c|c|c|c|c|c|c|c|c|c|c|c|c|}
\hline & \multicolumn{14}{|c|}{ MUESTRAS } \\
\hline ESPECIES & I & II & III & IV & $\mathbf{V}$ & VI & VII & VIII & IX & $X$ & XII & XIII & XIV & XV \\
\hline$\frac{A M M O N I A}{A . \text { beccarii }}$ & & & & & & & & & & & & & & \\
\hline A. beccarii & $5 \%$ & & & & $7 \%$ & $7,3 \%$ & & & $7 \%$ & & $24 \%$ & & & $\frac{5,3 \%}{8 \%}$ \\
\hline ANOMALINOIDES & & & & & & & & & & & & & & \\
\hline Anomalinoides sp & & $7 \%$ & & & & & & & & & & $29,7 \%$ & & \\
\hline ASTERIGERINATA & & & & & & & & & & & & & & \\
\hline A. planorbis & & $17,7 \%$ & $9,3 \%$ & $6,3 \%$ & $5,7 \%$ & $8 \%$ & & $5 \%$ & & & & & & \\
\hline ASTRONONION & & & & & & & & & & & & & & \\
\hline A. stelligerum & & & & & & & & & & & & $5 \%$ & & \\
\hline$\frac{{ }_{\text {BOLIVINA }}}{\text { B.pseudoplicata }}$ & & & & & & & $63 \%$ & & & & & & & \\
\hline $\begin{array}{l}\text { B. pseudoplicata } \\
\text { BRIZALINA }\end{array}$ & & & & & & & $6,3 \%$ & & & & & & & \\
\hline B. dilatata & & & & & & & & & & $8,3 \%$ & & & & \\
\hline BULIMINA & & & & & & & & & & & & & & \\
\hline B. lappa & & & & & & & & $6,7 \%$ & & $16 \%$ & & & & \\
\hline CASSIDULINA & & & & & & & & & & & & & & \\
\hline C. neocarinata & $45,7 \%$ & $14,3 \%$ & & $14,3 \%$ & & & & $6,3 \%$ & & $9 \%$ & & & & $8,7 \%$ \\
\hline CIBICIDES & & & & & & & & & & & & & & \\
\hline C. lobatulus & & & $5 \%$ & $5 \%$ & $7 \%$ & & $\frac{7,3 \%}{16 \%}$ & $6 \%$ & \begin{tabular}{l|l}
$5,3 \%$ \\
$147 \%$
\end{tabular} & & & & & $73 \%$ \\
\hline C. refulgens & & & $5 \%$ & & $5,3 \%$ & & $16 \%$ & $13 \%$ & & & & & $16 \%$ & $7,3 \%$ \\
\hline $\begin{array}{l}\text { CRIBOELPHIDIUM } \\
\text { C. decipiens }\end{array}$ & & & & & & & & & & & $6 \%$ & & $21 \%$ & $8,3 \%$ \\
\hline ELPHIDIUM & & & & & & & & & & & & & & \\
\hline E. advenum & & & $14 \%$ & & & & & & & & & & & \\
\hline E. crispum & & & & & $8,7 \%$ & $24,3 \%$ & $7,3 \%$ & $11 \%$ & $13,3 \%$ & $6,7 \%$ & $6,3 \%$ & & & $6,3 \%$ \\
\hline E. macellum & & & & & & & & & & & & & $5,3 \%$ & \\
\hline Elphidium $\mathrm{sp}$ & & & & & & & & & & & & & $6,7 \%$ & \\
\hline HANZAWAIA & & & & & & & & & & & & & & \\
\hline H. boueana & & & $6,7 \%$ & $5,3 \%$ & $7 \%$ & & & & & & & & & \\
\hline HETEROLEPA & & & & & & & & & & & & & & \\
\hline H. bellincionii & & $5,3 \%$ & $6 \%$ & & & & & $7,7 \%$ & $8,7 \%$ & & & & & \\
\hline HOPKINSINA & & & & & & & & & & & & & & \\
\hline H. bononiensis & & & & & & & & & & $7,7 \%$ & & & & \\
\hline \begin{tabular}{|l} 
LENTICULINA \\
\end{tabular} & & & & & & & & & & & & & & \\
\hline L. calcar & & & & $7 \%$ & & & & & & & & & & \\
\hline NONION & & & & & & & & & & & & & & \\
\hline N. asterizans & $12,3 \%$ & $8,7 \%$ & $11 \%$ & & $9 \%$ & $24,7 \%$ & $6,7 \%$ & $6,7 \%$ & $8,7 \%$ & $5,7 \%$ & $46,7 \%$ & $39,3 \%$ & & $30,7 \%$ \\
\hline
\end{tabular}

Tabla 1. Porcentaje de abundancia de las especies representadas por más del $5 \%$ en cada muestra.

Relative abundance (in percentage) of the species represented by more than $5 \%$ of the total assemblage in each sample.

manifiesto en las curvas de heterogeneidad y equitatividad. Estos parámetros, por el contrario, muestran un aumento importante en la base de la sección (de la muestra I a la II), que es seguido de una tendencia aproximadamente plana hasta la muestra V. Asimismo, en la curva de la equitatividad, el máximo se alcanza en la muestra V, no en la IV (Fig. 2).

A partir de la muestra IV se asiste a un importante descenso en el número de especies hasta la muestra VI, donde se detecta el valor más bajo. En las curvas de equitatividad y de diversidad de Shannon-Wiener se constata el mínimo de la muestra VI, sin embargo, los valores más bajos de diversidad según estos parámetros no se alcanzan aquí, sino en la muestra XII.

Tras un incremento de la diversidad en la muestra VII, hasta alcanzar valores similares a los anteriores, se produce un nuevo descenso hasta la muestra X. No obstante, en este caso, la caída experimentada por la diversidad no es tan drástica como la comentada anteriormente. Tanto el ascenso brusco como la ligera disminución de la diversidad se detecta de igual forma en todas las curvas obtenidas (Fig. 2).

Finalmente, desde la muestra XII hasta la XIV se produce un nuevo incremento progresivo de la diversidad, que es seguido de otro descenso hasta el final de la sección. Este patrón se observa en todas las curvas.

\section{RELACIÓN P/P+B}

A lo largo de toda la sección puede observarse que la proporción de foraminíferos bentónicos es siempre superior a la de planctónicos, excepto en la muestra VII, donde esta relación se invierte (Fig. 2).

En general, la relación $\mathrm{P} / \mathrm{P}+\mathrm{B}$ disminuye de muro a techo de la sección, aunque esta tendencia se ve interrumpida por la existencia de dos picos, uno en la muestra VII y otro en la XIV (Fig. 2). En detalle, el porcentaje de foraminíferos planctónicos disminuye ligeramente de forma progresiva desde la base de la sección $(43 \%)$ hasta la muestra V $(25 \%)$. Tras un pequeño incremento en la muestra VI $(27 \%)$, la relación $\mathrm{P} / \mathrm{P}+\mathrm{B}$ sufre un brusco incremento, llegándose a alcanzar un $60 \%$ en la siguiente muestra (muestra VII). El porcentaje de foraminíferos planctónicos decrece drásticamente a partir de esta muestra hasta la muestra $\mathrm{X}$, pasándose desde un $60 \%$ hasta un $0,3 \%$. 

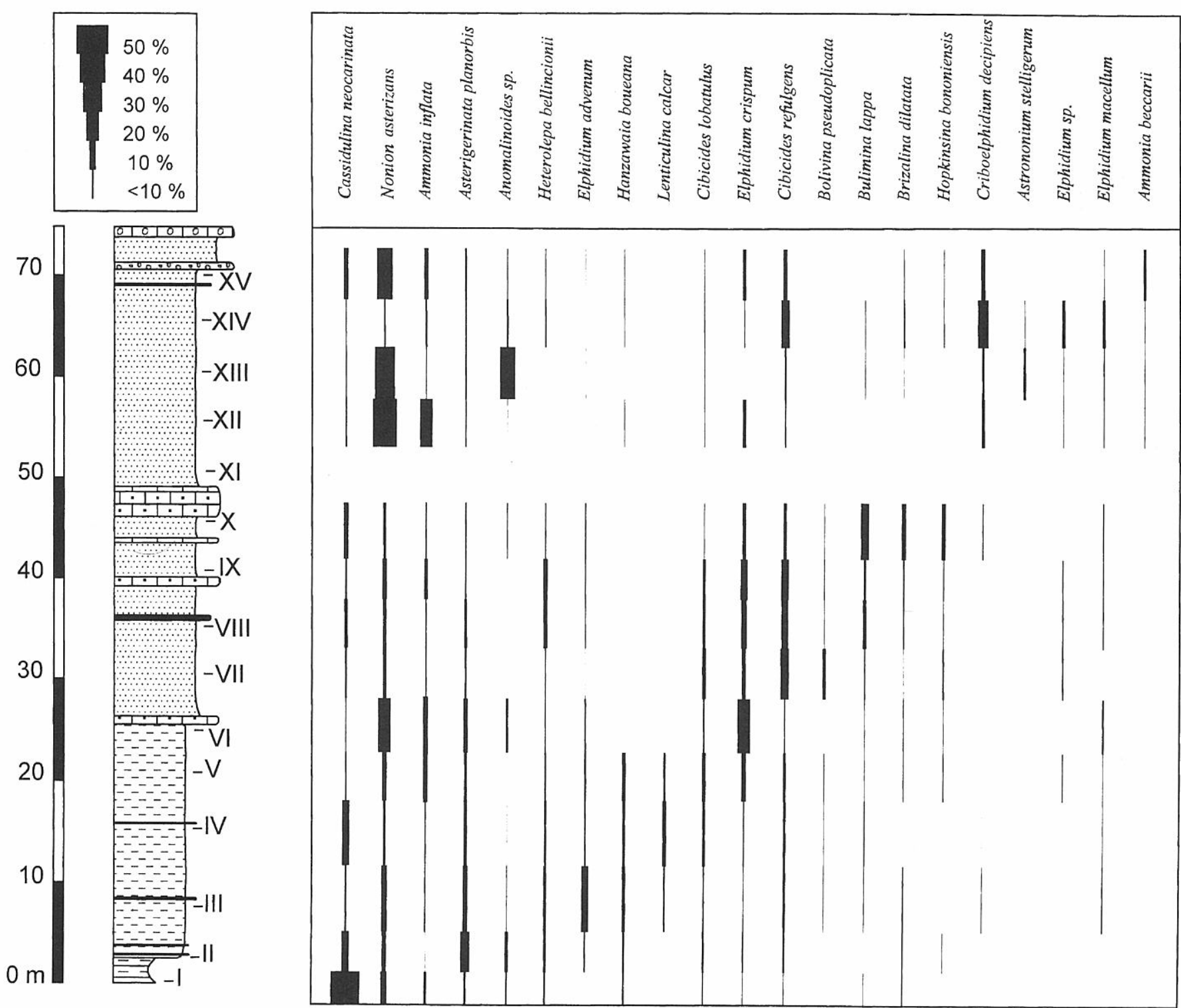

Figura 3. Distribución de abundancia de las especies de foraminíferos bentónicos representadas en más del 5\% del total de la asociación en cada muestra.

Abundance of benthic foraminifer species represented by more than $5 \%$ of the total assemblage in each sample.

En la mitad superior de la sección los valores de la relación P/P+B están por debajo de los alcanzados en la mitad inferior de la sección. No obstante, también se detecta un pico en la proporción de foraminíferos planctónicos en la muestra XIV de un $21 \%$.

\section{ASOCIACIONES DE FORAMINÍFEROS BENTÓNICOS}

\section{Distribución de abundancias}

En la Fig. 3 se muestra la distribución de las especies de foraminíferos bentónicos más abundantes (ver también Tabla 1). Como se observa en esta figura, las margas de la base de la sección están dominadas por Cassidulina neocarinata Thalmann, que representa casi el 50\% de la asociación. Nonion asterizans Fitchel y Moll (= Florilus boueanum) y Ammonia inflata (Linneo) son otras especies abundantes en la asociación de esta muestra (Tabla 1).

En los limos, desde la muestra II a la VI, se encuentran asociaciones muy diversas, representadas por un gran número de especies que sobrepasan el $5 \%$ en abundancia. Las especies más representativas en este tramo de la sección son Asterigerinata planorbis (d'Orbigny), Cassidulina neocarinata y Nonion asterizans, seguidas de distintas especies de Cibicides y Elphidium (Tabla 1). Cabe destacar la presencia de Lenticulina calcar (Linneo) y Hanzawaia boueana (d'Orbigny) como especies restringidas a esta litología ( $H$. boueana se encuentra de forma casi anecdótica en la parte alta de la sección, Fig. 3).

En el tramo de arenas finas-medias, representado en las muestras VII a IX, las especies de foraminíferos 
a)

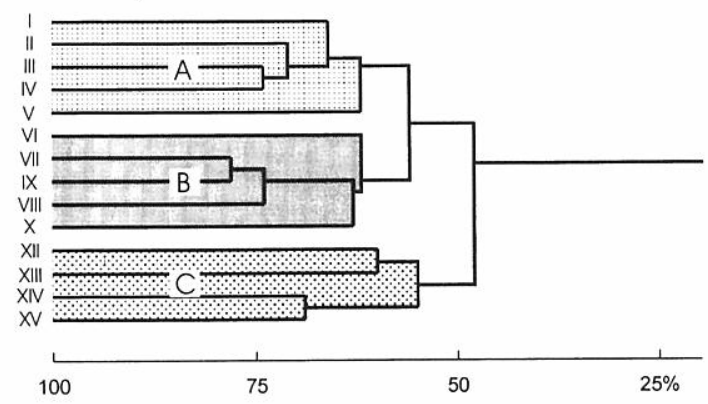

b)

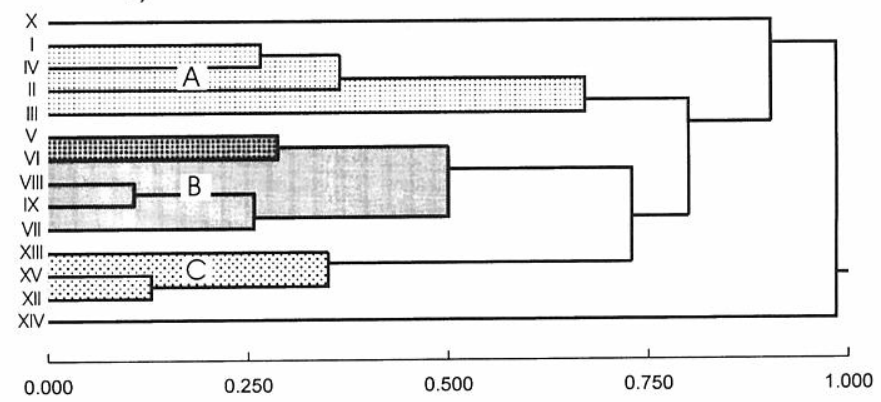

Figura 4. a) Análisis “cluster" binario, donde se evalúa de forma cualitativa la similitud entre pares de muestras, considerando exclusivamente presencia $(=1)$ y ausencia $(=0)$ de especies. El índice de similitud usado es el índice de Sorenson; b) análisis "cluster" cuantitativo en modo "Q", que tiene en cuenta la abundancia relativa de las diferentes especies. Se ha construido el "cluster" con el método de encadenamiento medio ("average linkage") y como índice de similitud entre muestras se ha usado el índice de correlación de Pearson.

a) Binary cluster analysis to show the similarity between samples, considering presence $(=1)$ and absence $(=0)$ of species exclusively. We have used the Sorensen index as the similarity index; b) $Q$-mode cluster analysis, considering the relative abundance of each species. We have used the average-linkage method and the Pearson correlation index.

bentónicos más abundantes son Elphidium crispum y distintas especies de Cibicides. Otras especies subordinadas son Heterolepa bellincionii (Gianninie y Tavani), Nonion asterizans, Cassidulina neocarinata, Bolivina pseudoplicata Heron-Allen y Earland, Bulimina lappa (Cushman y Parker) y Asterigerinata planorbis (Tabla 1).

En la base de las arenas gruesas (muestra $X$ ) se reconoce una asociación de foraminíferos bentónicos diferente a las reconocidas en el resto de la sección. Esta muestra está caracterizada por diversas especies de la superfamilia Buliminacea [Bulimina lappa, Brizalina dilatata (Reuss) y Hopkinsina bononiensis (Fornasini)] y por Cassidulina neocarinata (Tabla 1).

En la parte alta de la sección (arenas gruesas y conglomerados) se reconocen los máximos de abundancia de Nonion asterizans (Fig. 3). Otros organismos representativos son Ammonia inflata, Cribroelphidium decipiens (Costa), Anomalinoides sp. y distintas especies de Cibicides y Elphidium (Tabla 1). Es de destacar que Astrononium stelligerum (d'Orbigny) y Ammonia beccarii (Linneo) son dos especies que están restringidas a este tramo de la sección. Dentro de este tramo litológico, la muestra XIV supone una anomalía. Aquí se detecta una substancial disminución en la abundancia de $N$. asterizans, A. inflata y E. crispum, mientras que Cibicides refulgens Montfort, Cribroelphidium decipiens y Elphidium macellum (Fichtel y Moll) experimentan un notable incremento (Fig. 3).

\section{Análisis multivariante}

El análisis "cluster" binario usando el índice de Sorenson agrupa aquellas muestras con un mayor número de especies comunes. Los resultados obtenidos de este análisis se muestran en la Fig. 4a, donde se pueden reconocer tres agrupaciones ("clusters"): una primera, Grupo A, formada por las cinco primeras muestras; una segunda, Grupo B, que va desde la muestra VI hasta la muestra X; y, una tercera agrupación, Grupo C, que está constituida por las muestras XII, XIII, XIV y XV. Esta sucesión de las agrupaciones conlleva cambios en las asociaciones que quedan reflejados en la distribución de especies más abundantes (Fig. 3). Así, las muestras correspondientes al Grupo A están dominadas por Cassidulina neocarinata, Asterigerinata planorbis, Elphidium advenum (Cushman), Hanzawaia boueana y Lenticulina calcar. Las asociaciones del Grupo B, por su parte, están dominadas por Elphidium crispum, Cibicides refulgens, C. lobatulus (Walker y Jacob) y Heterolepa bellincionii. Finalmente, las asociaciones de la parte alta de la sección, correspondientes al Grupo C, están claramente caracterizadas por Nonion asterizans, Ammonia inflata, Anomalinoides sp. y Cribroelphidium decipiens.

Estas tres agrupaciones muestran una clara correlación entre asociaciones de foraminíferos bentónicos y litología. Así, la primera agrupación representa las margas y los limos margosos de la parte baja de la sección. La segunda incluye las arenas finas y medias con intercalaciones de tempestitas distales de la parte central de la sección. Por encima, las arenas gruesas y los conglomerados del techo están caracterizados por la tercera agrupación.

El análisis "cluster" cuantitativo en modo "Q" (Fig. 4b) ayuda a diferenciar varios subgrupos, relacionados con las agrupaciones anteriormente reconocidas, cuya similitud está caracterizada por la correlación de la abundancia de las especies presentes en cada muestra. Dentro del Grupo A definido anteriormente (Fig. 4a), las muestras I y IV están estrechamente relacionadas, ya que comparten aproximadamente las mismas especies y, además, están dominadas por Cassidulina neocarinata (Fig. 3). Por su parte, las muestras II y III se relacionan con el par anterior pero a más distancia, ya que la abundancia de las especies difiere substancialmente con 
relación a las muestras I y IV. En este grupo también es común, y en ocasiones muy abundante, Asterigerinata planorbis, como en la muestra II.

En el "cluster" binario la muestra V se incluye en el Grupo A, mientras que en el "cluster" cuantitativo está relacionada con la muestra VI (Fig. 4b), perteneciente al Grupo B de la Figura 4a. Esta mayor similitud con las muestras del Grupo B es debida a que, aunque en la muestra $\mathrm{V}$ están presentes especies comunes con el Grupo A, las especies más abundantes en esta muestra son también las más abundantes en la muestra VI (Fig. 3). Así, $C$. neocarinata se encuentra de forma casi anecdótica en la muestra V, mientras que Nonion asterizans, Ammonia inflata y Elphidium crispum, especies poco representadas en el Grupo A, dominan en la muestra VI (Fig. 3). Esta distribución de abundancias de especies condiciona la covarianza entre las muestras V y VI, quedando estrechamente relacionadas en el "cluster" cuantitativo (Fig. 4b).

Otro subgrupo dentro del Grupo B es el representado por las muestras VII, VIII y IX, caracterizadas por el dominio de Cibicides refulgens, C. lobatulus y Elphidium crispum. Dentro de este subgrupo, las muestras VIII y IX, donde también domina Heterolepa bellincionii, muestran el mayor grado de similitud (Fig. 4b). La muestra VII se separa del par anterior, ya que numerosas especies presentes en las muestras anteriores están ausentes en esta muestra (Fig. 3).

La muestra X, incluida en el Grupo B del análisis binario (Fig. 4a), se separa como una muestra independiente en el análisis cuantitativo (Fig. 4b). Esta falta de similitud se debe a que la muestra X está caracterizada por un conjunto de especies que están en muy baja proporción, o ausentes, en el resto de la sección (Tabla 1).

En el Grupo C, se unen las muestras XII, XIII y XV en función del índice de similitud de Pearson (Fig. 4b). Estas muestras están dominadas por Nonion asterizans, Ammonia inflata y Elphidium crispum. La muestra XIV, por su parte, tiene similitud con las anteriores en el número de especies presentes/ausentes, lo que determina su pertenencia al Grupo C (Fig. 4a). No obstante, no presenta similitud en el índice de Pearson porque la asociación de foraminíferos bentónicos de esta muestra está dominada por Cribroelphidium decipiens, Elphidium macellum y Cibicides refulgens, especies poco o nada representadas en las otras muestras. Paralelamente, las especies dominantes en las otras muestras del Grupo C experimentan una disminución muy notable en la abundancia en la muestra XIV (Fig. 3). Por todo ello, esta muestra permanece como muestra independiente en el "cluster" cuantitativo (Fig. 4b).

\section{DISCUSIÓN}

Las tres agrupaciones diferenciadas con el índice de Sorenson (Fig. 4a) sugieren un relevo de asociaciones de foraminíferos bentónicos relacionado con una somerización de la cuenca. Cassidulina neocarinata, especie que domina en las muestras del Grupo A, en la actualidad muestra los máximos de abundancia en ambientes de borde de plataforma externa y talud superior y suele estar asociada a condiciones ligeramente disaeróbicas (>0,5 ml/ $\mathrm{O}_{2}$ ) (Mullins et al., 1985).

En cuanto a los componentes mayoritarios del Grupo B, Elphidium crispum, Cibicides refulgens y Cibicides lobatulus, en la actualidad son componentes comunes en las praderas de Posidonia del Mediterráneo (Langer, 1988). Las praderas de Posidonia se distribuyen a lo largo de la franja submareal, alcanzando una profundidad media de 50 m (Pérès, 1989; Ros et al., 1989). Por tanto, la asociación que caracteriza el Grupo B se puede atribuir a un ambiente de plataforma media. Dentro de esta asociación, $C$. refulgens y $C$. lobatulus muestran un rango de distribución más amplio que E. crispum. Esto es debido al modo de vida y a los hábitos alimenticios. Elphidium crispum es epifítico y vive suspendido por sus rizópodos, mientras que Cibicides refulgens y $C$. lobatulus se encuentran permanentemente fijas a un substrato (hojas de algas, conchas de bivalvos, etc.) pastoreando e incluso, en el caso de C. refulgens, parasitando a bivalvos (Alexander y DeLaca, 1987; Goldstein, 1999).

Finalmente, la presencia conjunta de Nonion asterizans, Ammonia inflata, Anomalinoides sp. y Cribroelphidium decipiens como especies dominantes en el Grupo C denota el carácter somero de estas muestras. Las asociaciones dominadas por estas especies se encuentran preferentemente en ambientes de plataforma interna somera, en un rango batimétrico de 20-30 m de profundidad (por ejemplo, Sen Gupta, 1980; Murray, 1991). Nonion asterizans y Ammonia inflata son dos especies infáunicas oportunistas (Yanko et al., 1999), tolerantes a grandes variaciones en el medio. En medios someros suelen producirse fluctuaciones importantes en parámetros tales como turbidez, turbulencia del agua y/o salinidad, lo que favorece a dichas especies frente a otras con requerimientos ecológicos más estrictos.

La progresiva somerización reflejada mediante las asociaciones de foraminíferos bentónicos es congruente con los datos obtenidos del estudio de las facies, de las asociaciones de pectínidos y de las señales tafonómicas observadas en la macrofauna (Aguirre, 1996, 1998; Aguirre et al., 1996). La generalizada disminución hacia el techo de la sección de la relación P/P+B (Fig. 2), al igual que el aumento en el grado de destrucción tafonómica de las conchas, es coherente con la somerización inferida anteriormente.

Considerando los rangos batimétricos máximos en los que coinciden las especies más abundantes $(>5 \%)$ de cada muestra (Fig. 5) se pueden reconocer dos ciclo menores de somerización limitados por un pulso de profundización. El primer ciclo se extiende desde la base de la sección hasta la muestra VI y el segundo desde la muestra VII hasta el techo de la sección. En el primer ciclo se asiste a una disminución en el rango de distribución batimétrica de las asociaciones, pasándose de asociaciones que se distribuyen de 30 a $100 \mathrm{~m}$ de profundidad a asociaciones que se desarrollan 


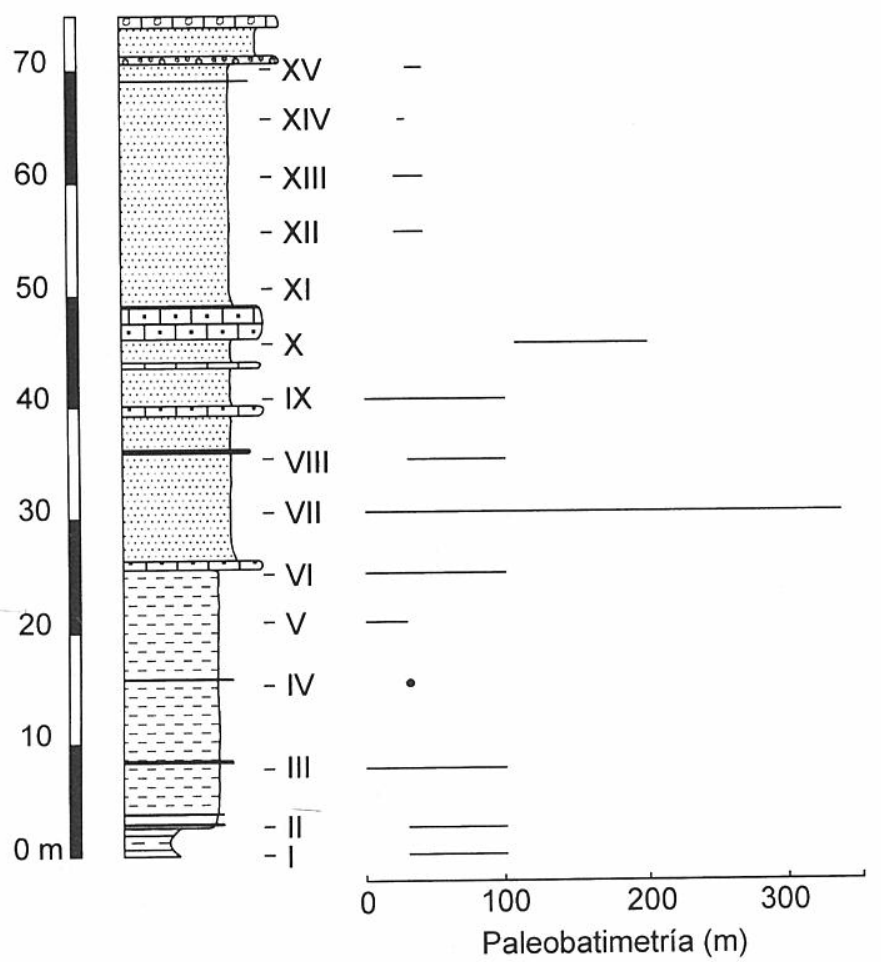

Figura 5. Profundidades inferidas para los depósitos de la sección Palmo de Salas según las asociaciones de foraminíferos bentónicos. Las líneas horizontales representan los rangos batimétricos máximos según la distribución batimétrica de las mismas especies en el Mediterráneo actual. En general, cabe destacar la tendencia a la somerización desde la base de la sección hasta el techo.

Inferred depth for each sample based on the depth distribution of the benthic foraminifer assemblages. The horizontal lines represent the maximum bathimetric ranges of the species in the present-day Mediterranean. An upward shallowing trend is observable.

preferentemente por debajo de $30 \mathrm{~m}$ de profundidad (Fig. 5). El mismo patrón se observa en el segundo ciclo reconocido (Fig. 5). La tendencia a la somerización en ambos ciclos también se ve constatada por la disminución en la proporción de foraminíferos planctónicos frente a bentónicos (Fig. 2).

El límite entre los dos ciclos está marcado por un sustancial incremento en el rango batimétrico de las especies de la muestra VII con respecto a los de las muestras V y VI (Fig. 5), que se correlaciona con un cambio en las asociaciones de foraminíferos bentónicos. Así, el techo del primer ciclo de somerización está dominado por especies típicas de plataforma interna somera (Nonion asterizans, Ammonia inflata y Elphidium crispum. Tabla 1). Por el contrario, la muestra VII está dominada por Cibicides refulgens, C. lobatulus, Elphidium crispum, Nonion asterizans y Bolivina pseudoplicata (Tabla 1) que se desarrollan preferentemente en ambientes de plataforma mediaexterna (Murray, 1991). La profundización reflejada en el cambio entre los dos ciclos reconocidos también se pone de manifiesto por el notable incremento de la relación $\mathrm{P} / \mathrm{P}+\mathrm{B}$, alcanzándose el máximo en la muestra VII (Fig. 2).

En definitiva, las variaciones en las asociaciones de foraminíferos bentónicos, junto con los rangos batimétricos que se infieren de las mismas, y la relación $\mathrm{P} / \mathrm{P}+\mathrm{B}$ parecen reflejar variaciones del nivel del mar a lo largo del depósito de los materiales estudiados. Por un lado, la somerización generalizada detectada desde la base hacia el techo de la sección puede correlacionarse con la bajada generalizada del nivel del mar que tuvo lugar durante todo el Plioceno inferior (por ejemplo, Haq et al., 1987; Hardenbol et al., 1998). Por otro lado, los dos ciclos menores reconocidos pueden corresponderse con alguna de las variaciones del nivel del mar de mayor frecuencia detectadas durante este periodo de tiempo, tanto a nivel global (Poag y Low, 1985; Sprovieri, 1990; Kranz, 1991; Zubakov, 1992; Thiede et al., 1998) como en otras cuencas del litoral S de España (Aguirre, 1995, 2000).

Según la distribución batimétrica de las especies, el rango de profundidad obtenido para la muestra VII es incluso superior al reconocido en las margas de la base, muestra I (Fig. 5). Sin embargo, la litología, las facies y las asociaciones de macrofauna indican que la situación de nivel del mar supuesta para la muestra VII no debió superar a la de la muestra I, incluso aunque la relación $\mathrm{P} / \mathrm{P}+\mathrm{B}$ sea significativamente mayor en las muestras VII y VIII que en la I. Por tanto, el importante incremento de la relación $\mathrm{P} / \mathrm{P}+\mathrm{B}$ en el límite entre los dos ciclos parece estar controlado por otros factores ambientales, además de la posible influencia del ascenso del nivel del mar.

Una posible causa del aumento en la relación $\mathrm{P} / \mathrm{P}+\mathrm{B}$ es la existencia de corrientes cargadas de nutrientes. Dichas corrientes generarían una mayor producción primaria en las aguas superficiales y, como consecuencia, un incremento en las poblaciones de foraminíferos planctónicos que se alimentan del fitoplancton (por ejemplo, Summerhayes et al., 1992; Martinez et al., 1999). La entrada masiva de nutrientes, por ejemplo por afloramientos costeros de aguas frías profundas (corrientes de "upwelling"), usualmente conlleva la generación de fondos empobrecidos en oxígeno. Estas condiciones favorecen el desarrollo de asociaciones de foraminíferos bentónicos poco diversas, caracterizadas por muchos individuos de algunas especies oportunistas (Sen Gupta y Machain-Castillo, 1993; Murray, 1995; Kaiho, 1994, 1999; Loubere y Fariduddin, 1999). Sin embargo, los valores de diversidad de la muestra VII son similares a los de las muestras infra y suprayacentes (Fig. 2).

Otra posibilidad es la entrada de nutrientes desde el continente. No obstante, las aguas cargadas en nutrientes procedentes del continente generarían una cuña de aguas superficiales salobres, o incluso dulces. Esta situación inhibiría el desarrollo de las comunidades de foraminíferos planctónicos, situación que es contraria al incremento de planctónicos que se produce en la muestra VII. 
Una explicación alternativa es que exista una mayor influencia en la región estudiada de aguas superficiales ricas en nutrientes procedentes del Atlántico. Estas aguas tienen un mayor contenido en nutrientes que las del Mediterráneo (De Rijk et al., 1999), por lo que su influencia pudo favorecer el desarrollo de poblaciones de foraminíferos planctónicos. Si bien las aguas atlánticas son más productivas que las mediterráneas, no están lo suficientemente cargadas de nutrientes como para generar fondos muy empobrecidos en oxígeno y afectar a la diversidad de las comunidades de foraminíferos bentónicos.

En conclusión, el pico en la relación $\mathrm{P} / \mathrm{P}+\mathrm{B}$ de la muestra VII pudo estar condicionado por el efecto combinado de un aumento relativo del nivel del mar junto con una entrada de aguas ricas en nutrientes procedentes del Atlántico hacia las regiones más septentrionales de la cuenca de Almería-Níjar (ver paleogeografía en Aguirre, 1998). Este segundo proceso amplificaría la señal en la relación $\mathrm{P} / \mathrm{P}+\mathrm{B}$ más de lo que pudiera reflejar exclusivamente un aumento del nivel del mar.

Dentro del ciclo de somerización inferior se aprecia un pico en la abundancia de especies en la muestra IV, aunque no se corresponde con ningún cambio en los índices de heterogeneidad y equitatividad (Fig. 2). Esto implica que, aunque el número de especies es mayor, la asociación está dominada por sólo unas pocas especies. Al igual que ocurre en la muestra I, Cassidulina neocarinata es la especie más abundante de la asociación en la muestra IV (Tabla 1). El dominio de esta especie en las dos muestras queda puesto de manifiesto en el alto grado de similitud por el análisis "cluster" cuantitativo (Fig. 4b). C. neocarinata, además de ser una especie capaz de desarrollarse en ambientes disaeróbicos, como se comentó anteriormente, también resiste ambientes con un cierto grado de turbidez (Mullins et al., 1985). En este sentido, la progradación de los sedimentos del frente deltaico del Abrioja debió jugar un papel fundamental. La progresiva somerización relacionada con el primer ciclo tendría asociada una mayor influencia de los lodos procedentes de las descargas deltaicas en ambientes cada vez más someros y, por tanto, un incremento en el grado de turbidez de las aguas.

En el ciclo superior, las muestras X y XIV, incluidas dentro de los Grupos B y C, respectivamente, del "cluster" cualitativo de Sorenson, se individualizan como muestras independientes en el "cluster" cuantitativo (Fig. 4). La muestra X, por su parte, está caracterizada por una asociación de foraminíferos bentónicos particular: Bulimina lappa, Cassidulina neocarinata, Brizalina dilatata y Hopkinsina bononiensis (Tabla 1). Esta asociación de especies sugiere un fondo disaeróbico (Sen Gupta y Machain-Castillo, 1993; Kaiho, 1994, 1999). No obstante, estas condiciones son poco frecuentes en un medio somero, como el que se ha supuesto para el depósito de las arenas y conglomerados de la parte alta de la sección: frente deltaico afectado por las tormentas (Aguirre, 1995, 1996, 1998; Aguirre et al., 1996) con un mínimo en la relación $\mathrm{P} / \mathrm{P}+\mathrm{B}$ (Fig. 2). Esta aparente incongruencia podría explicarse por un aumento relativo en la tasa de sedimentación. Una mayor proximidad a la desembocadura de los canales distributarios del delta favorecería el enterramiento rápido de la materia orgánica antes de ser oxidada. Por tanto, la oxidación de la materia orgánica ocurriría dentro de la columna de sedimento consumiéndose gran parte del oxígeno disuelto en las aguas intersticiales. De esta manera, se generan sedimentos empobrecidos en $\mathrm{O}_{2}$ y una proliferación de especies de foraminíferos tolerantes a estas condiciones frente al resto (Sen Gupta y Machain-Castillo, 1993; Kaiho, 1994, 1999).

La batimetría calculada para la muestra $X$ a partir de las especies dominantes es mayor que la que se infiere del resto de las evidencias sedimentológicas y paleontológicas de los propios sedimentos. Esto es debido a que la asociación responde más a condiciones ecológicas del medio que a rangos batimétricos. Aunque dichas condiciones son más típicas de medios de plataforma media-externa (Schmiedl et al., 1997), en determinadas condiciones, como las explicadas anteriormente, puede imponerse en medios más someros.

En el caso de la muestra XIV se produce un cambio significativo en las especies dominantes con relación a las muestras XII, XIII y XV. Además de los cambios en las proporciones de las especies mayoritarias, la muestra XIV es anómala dentro del Grupo C, ya que también se detectan picos en la diversidad y en la relación $\mathrm{P} / \mathrm{P}+\mathrm{B}$ (Fig. 2). Todos estos cambios asociados a esta muestra pueden explicarse por dos mecanismos que no son excluyentes entre sí. Por un lado, un máximo relativo en la relación $\mathrm{P} / \mathrm{P}+\mathrm{B}$ podría estar conectado con un aumento en la columna de agua. En relación con esta hipótesis, la asociación de foraminíferos bentónicos para esta muestra está representada por los géneros Cribroelphidium, Cibicides y Elphidium, considerados como formas epifíticas (Langer, 1988, 1993). Como consecuencia, esta asociación podría indicarnos el desarrollo de vegetación en el fondo durante una etapa de escasa o nula (?) sedimentación deltaica, hecho que se ve favorecido durante ascensos del nivel del mar (Loutit et al., 1988).

Por otro lado, al igual que se argumentaba para el máximo de la muestra VII (Fig. 2), el pico de diversidad de la muestra XIV podría estar relacionado con un aumento en el contenido de nutrientes en la cuenca.

En conclusión, los valores anómalos de la muestra XIV parecen estar conectados con una serie de fenómenos que pudieron actuar conjuntamente. Discernir entre una subida relativa del nivel del mar y el aporte de nutrientes a la cuenca no es fácil, ya que no existen argumentos definitivos para ninguna de ambas alternativas. Por tanto, en este trabajo proponemos ambas hipótesis como explicaciones alternativas y complementarias, no excluyentes.

\section{CONCLUSIONES}

1.- La secuencia de edad Plioceno inferior del Palmo de Salas, N de la cuenca de Almería-Níjar (SE de España), representa una secuencia somerizante hacia el 
techo, donde se pasa de depósitos de plataforma externa a medios de plataforma interna somera. En los sedimentos más profundos domina Cassidulina neocarinata, mientras que las asociaciones de la zona más somera de la sección están caracterizadas por los géneros Ammonia, Nonion, Cribroelphidium y Elphidium. Esta tendencia a la somerización también se pone de manifiesto por un generalizado descenso de la relación planctónicos/ bentónicos $(\mathrm{P} / \mathrm{P}+\mathrm{B})$.

2.- Un análisis "cluster" binario de Sorenson muestra la existencia de tres agrupaciones que se relacionan claramente con la litología: Grupo A en las margas y limos; Grupo B en limos y arenas finas con intercalación de tempestitas distales; y, Grupo $\mathrm{C}$ en arenas y conglomerados con tempestitas proximales. Este relevo en las asociaciones refleja la dependencia para determinadas especies de foraminíferos bentónicos del tipo de substrato. Dicho cambio litológico, además, está controlado por la somerización de la cuenca hacia el techo.

3.- Dentro de la tendencia de somerización se han reconocido dos ciclos de somerización de mayor frecuencia. Las seis primeras muestras corresponden al ciclo inferior. A partir de la muestra VII comienza el segundo ciclo hasta el techo de la sección. Cada uno de estos ciclos se deduce de los cambios experimentados en las asociaciones de especies, así como de variaciones en la diversidad y en la relación $\mathrm{P} / \mathrm{P}+\mathrm{B}$.

4.- El cambio entre los dos ciclos de somerización se confirma mediante un análisis "cluster" cuantitativo en modo "Q", de forma que las muestras del techo del primer ciclo (muestras V y VI), dominadas por las formas someras Nonion asterizans, Elphidium y Ammonia, se agrupan con un alto índice de similitud. Por su parte, la muestra VII se une con las muestras VIII y IX, caracterizadas por una asociación más típica de plataforma media (Cibicides spp., Elphidium spp., Heterolepa bellincionii, Nonion asterizans, Bolivina pseudoplicata y Bulimina lappa).

5.- El "cluster" cuantitativo, además de diferenciar asociaciones según la distribución batimétrica, discrimina de forma clara asociaciones de foraminíferos bentónicos que están controladas por las características del fondo. Por un lado, se diferencia una asociación típica de fondos disaeróbicos (muestra X), que está representada por bulimináceos (Bulimina lappa, Brizalina dilatata y Hopkinsina bononiensis) y Cassidulina neocarinata. Por otro lado, se individualiza una asociación dominada por formas epifíticas (muestra XIV), como son Cibicides, Elphidium y Cribroelphidium.

6.- Dentro del ciclo de somerización superior, la muestra XIV supone una anomalía. Esta muestra se separa del resto en el "cluster" cuantitavo (debido a un cambio en las proporciones de las especies dominantes) y, además, experimenta un incremento de la relación $\mathrm{P} / \mathrm{P}+\mathrm{B}$ y de la diversidad. Este pico puede explicarse tanto por un ligero aumento del nivel del mar, junto con el desarrollo de una densa cobertera de algas en el fondo, como por una entrada de nutrientes a la cuenca. Ambas alternativas no son excluyentes, sino complementarias.

\section{AGRADECIMIENTOS}

Quisiéramos agradecer los comentarios y correcciones de dos revisores anónimos, que, sin duda, han contribuido de forma decisiva a la mejora del trabajo. Este trabajo ha sido financiado por el Proyecto de Investigación "Cambios climáticos en el Sur de España durante el Neógeno", subvencionado por la Fundación Ramón Areces, y por el Grupo de Investigación "Paleogeografía de cuencas sedimentarias", de la Junta de Andalucía.

\section{BIBLIOGRAFÍA}

Aguirre, J. 1995. Tafonomía y Evolución Sedimentaria del Plioceno Marino en el Litoral Sur de España entre Cádiz y Almería. Tesis Doctoral, Universidad de Granada, 419 pp. (inédita).

Aguirre, J. 1996. Modelo de tafofacies en el Plioceno inferior de la Cuenca de Almería-Níjar (SE de España). In: II Reunión de tafonomía y fosilización (Eds. G. Meléndez Hevia, M.F. Blasco Sancho e I. Pérez Urresti). Zaragoza, 11-18.

Aguirre, J. 1998. El Plioceno del SE de la Península Ibérica (provincia de Almería). Síntesis estratigráfica, sedimentaria, bioestratigráfica y paleogeográfica. Revista de la Sociedad Geológica de España, 11, 297-315.

Aguirre, J. (2000). Evolución paleoambiental y análisis secuencial de los depósitos pliocenos de Almayate (Málaga, S de España). Revista de la Sociedad Geológica de España, 13, 431-443.

Aguirre, J. and Jiménez, A.P. 1997. Census assemblages in hard-bottom coastal communities: A case study from the Plio-Pleistocene Mediterranean. Palaios, 12, 598-608.

Aguirre, J. and Jiménez, A.P. 1998. Fossil analogues of the present-day ahermatypic Cladocora caespitosa coral banks: Sedimentary setting, dwelling community, and taphonomy (Late Pliocene, W Mediterranean). Coral Reefs, 17, 203-213.

Aguirre, J. and Sánchez Almazo, I.M. 1998. Foraminiferal assemblages in Upper Pliocene deposits of Almería-Níjar Basin (SE Spain): Biostratigraphic and palaeoenvironmental significance. 15th International Sedimentological Congress, Universidad de Alicante, p. 120.

Aguirre, J., Braga, J.C., Jiménez, A.P. and Rivas, P. 1996. Substrate-related changes in pectinid fossil assemblages. Palaeogeography, Palaeoclimatology, Palaeoecology, 126, 291-308.

Alexander, S.P. and DeLaca, T.E. 1987. Feeding adaptations of the foraminiferan Cibicides refulgens living epizoically and parasitically on the Antarctic scallop Adamussium colbecki. Biological Bulletin, 173, 136-159.

Alve, E. and Murray, J.W. 1999. Marginal marine environments of the Skagerrak and Kattegat a baseline study of living (stained) benthic foraminiferal ecology. Palaeogeography, Palaeoclimatology, Palaeoecology, 146, 171-193.

Berger, W.H. 1979. Preservation of foraminifera. In: Foraminiferal ecology and paleoecology (Eds. J.H. Lipps, W.H. Berger, M.A. Buzas, R.G. Douglas and 
C.A. Ross). SEPM Short Course, Houston, Texas. No. 6 , 105-155.

Brett, C.E. 1995. Sequence stratigraphy, biostratigraphy, and taphonomy in shallow marine environments. Palaios, $\mathbf{1 0}$, 597-616.

Brett, C.E. and Baird, G.C. 1986. Comparative taphonomy: a key to paleoenvironmental interpretation based on fossil preservation. Palaios, 1, 207-227.

Davis, J.C. 1986. Statistics and Data Analysis in Geology. John Wiley \& Sons, Inc. (2 $2^{\mathrm{a}}$ edición), New York. 646 pp.

De Rijk, S. and Troelstra, S.R. 1997. Salt marsh foraminifera from the Great Marshes, Massachusetts: environmental controls. Palaeogeography, Palaeoclimatology, Palaeoecology, 130, 81-112.

De Rijk, S., Troelstra, S.R. and Rohling, E.J. 1999. Benthic foraminiferal distribution in the Mediterranean Sea. Journal of Foraminiferal Research, 29, 93-103.

Douglas, R.G. 1979. Benthic foraminiferal ecology and paleoecology: A review of concepts and methods. In: Foraminiferal Ecology and Paleoecology (Eds. J.H. Lipps, W.H. Berger, M.A. Buzas, R.G. Douglas and C.A. Ross). SEPM Short Course, Houston, Texas. No. 6 , 21-53.

Goldstein, S.T. 1999. Foraminifera: A biological overview. In: Modern Foraminifera (Ed. B.K. Sen Gupta). Kluwer Academic Publishers, 37-55.

Gooday, A.J. 1994. The biology of deep-sea foraminifera: A review of some advances and their applications in paleoceanography. Palaios, 9, 14-31.

Goy, J.L. y Zazo, C. 1982. Niveles marinos cuaternarios y su relación con la tectónica en el litoral de Almería (España). Boletín de la Real Sociedad de Historia Natural (Geología), 80, 171-184.

Haq, B.V., Hardenbol, J. and Vail, P.R. 1987. Chronology of fluctuating sea levels since the Triassic. Science, 235, 1156-1167.

Hardenbol, J., Thierry, J., Farley, M.B., Jacquin, T., Graciansky, P.C. and Vail, P.R. 1998. Mesozoic and Cenozoic sequence chronostratigraphic framework of European basins. In: Mesozoic and Cenozoic Sequence Stratigraphy of European Basins (Eds. P.C. Graciansky, J. Hardenbol, T. Jacquin, and P.R. Vail). Society of Economic Paleontologists and Mineralogists, Special Publication, 60, 3-13.

Jorissen, F.J. 1987. The distribution of benthic foraminifera in the Adriatic Sea. Marine Micropaleontology, 12, 21-48.

Kaiho, K. 1994. Benthic foraminiferal dissolved-oxygen index and dissolved-oxygen levels in the modern ocean. Geology, 22, 719-722.

Kaiho, K. 1999. Effect of organic carbon flux and dissolved oxygen on the benthic foraminiferal oxygen index (BFOI). Marine Micropaleontology, 37, 67-76.

Katz, M. E. and Thunell, R. C. 1984, Benthic foraminiferal biofacies associated with Middle Miocene to Early Pliocene oxygen-deficient conditions in the Eastern Mediterranean. Journal of Foraminiferal Research, 14, 187-202.

Kitazato, H. 1994. Foraminiferal microhabitats in four marine environments around Japan. Marine Micropaleontology, 24, 29-41.
Kranz, D.E. 1991. A chronology of Pliocene sea-level fluctuations: The U.S. middle Atlantic Coastal Plain record. Quaternary Science Review, 10, 163-174.

Langer, M.R. 1988. Recent epiphytic foraminifera from Vulcano (Mediterranean Sea). Revue de Paléobiologie, vol. Spéc. 2, 827-832.

Langer, M.R. 1993. Epiphytic foraminifera. Marine Micropaleontology, 20, 235-265.

Loubere, P. and Fariduddin, M. 1999. Benthic foraminifera and the flux of organic carbon to the seabed. In: Modern Foraminifera (Ed. B.K. Sen Gupta). Kluwer Academic Publishers, 181-199.

Loutit, T.S., Handerbold, J., Vail, P.R. and Baum, P. 1988. Condensed sections: the key to age dating and correlation of continental margin sequences. In: Sea-Level Changes: An Integrated Approach (Eds. C. Wilgus, B. Hastings, C. Ross, H. Posamentier, J. Van Wagoner and C.G.St.C. Kendall). Society of Economic Paleontologists and Mineralogists, Special Publication, 42, 183-213.

MacKensen, A., Schemiedl, G., Harloff, J. and Giese, M. 1995. Deep-sea foraminifera in the South Atlantic Ocean: Ecology and assemblage generation. Micropaleontology, 41, 342-358.

Magurran, A.E. 1989. Diversidad Ecológica y su Medición. Vedrá (trad. Española por A.M. Cirer), Barcelona. 200 pp.

Martinez, P., Bertrand, P., Shimmiedl, G.B., Cochrane, K., Jorissen, F.J., Foster, J. and Dignan, M. 1999. Upwelling intensity and ocean productivity changes off Cape Blanc (northwest Africa) during the last 70,000 years: geochemical and micropaleontological evidence. Marine Geology, 158, 57-74.

Miller, K.G. 1980. Cenozoic benthic foraminifera case histories of paleoceanographic and sea-level changes. In: Foraminifera. Notes for a Short Course (Eds. M.A. Buzas and B.K. Sen Gupta). University of Tennessee, Studies in Geology, No. 6, 107-126.

Montenat, C., Ott d'Estevou, Ph. et La Chapelle, G. 1990. Le bassin de Nijar-Carboneras et le couloir du basAndarax. Documents et Travaux IGAL, 12-13, 129-164.

Moodley, L. and Hess, C. 1992. Tolerance of infaunal benthic foraminifera for low and high oxygen concentration. Biological Bulletin, 183, 94-98.

Mullins, H.T., Thompson, J.B., McDouglas, K.Y. and Vercoutere, T.L. 1985. Oxygen-minimum zone edge effects: Evidence from the central California coastal upwelling system. Geology, 13, 491-494.

Murray, J.W. 1991. Ecology and Paleoecology of Benthic Foraminifera. Harlow, Essex, 397 pp.

Murray, J.W. 1995. Microfossil indicators of ocean water masses, circulation and climate. In: Marine Palaeoenvironmental Analysis from Fossils (Eds. D.W.J. Bosence and P.A. Allison). Geological Society Special Publication No. 83, 245-264.

Parker, F. L. 1958. Eastern Mediterranean foraminifera. Reports of the Swedish Deep-Sea Expedition, 8, 219-285.

Pérès, J.M. 1989. Historia de la biota mediterránea y la colonización de las profundidades. In: El Mediterráneo Occidental (Ed. R. Margalef). Omega, Barcelona, 200234. 
Pérez-Muñoz, A.B., Sánchez-Almazo, I.M. and Aguirre, J. 2000. Taphonomy and proximal-to-distal changes of benthic foraminifer assemblages in a glauconite-bearing bed (early Pliocene, Almería-Níjar Basin, SE Spain). The Second International Conference "Application of Microand Meioorganisms to environmental problems", Avalon, Canadá, 97-99.

Poag, C.W. and Low, D. 1985. Environmental trends among Neogene benthic foraminifers at Deep Sea Drilling Project Site 548, Irish continental margin. Initial Reports DSDP, 80, 489-503.

Postma, G. 1983. Water escape structures in the context of a depositional model of a mass flow dominated conglomeratic fan-delta (Abrioja Formation, Pliocene, Almería Basin, SE Spain). Sedimentology, 30, 91-103.

Ros, J.D., Romero, J., Ballesteros, E. y Gili, J.M. 1989. Buceando en aguas azules. El bentos. In: El Mediterráneo Occidental (Ed. R. Margalef). Omega, Barcelona, 235-297.

Schmiedl, G., Mackensen, A. and Müller, P.J. 1997. Recent benthic foraminifera from the eastern South Atlantic Ocean: Dependence on food supply and water masses. Marine Micropaleontology, 32, 249-287.

Sen Gupta, B.K. 1980. Ecology of benthic foraminifera. In: Foraminifera. Notes for a Short Course (Eds. M.A. Buzas and B.K. Sen Gupta). University of Tennessee, Studies in Geology, No. 6, 37-50.

Sen Gupta, B.K. and Machain-Castillo, M.L. 1993. Benthic foraminifera in oxygen-poor habitats. Marine Micropaleontology, 20, 183-201.

Serrano, F. 1990. El Mioceno medio en el área de Níjar (Almería, España). Revista de la Sociedad Geológica de
España, 3, 65-77.

Shi, G.R. 1993. Multivariate data analysis in palaeoecology and palaeobiogeography - a review. Palaeogeography, Palaeoclimatology, Palaeoecology, 105, 199-234.

Sprovieri, R. 1990. Plio-Pleistocene paleoclimatic evolution at ODP Leg 107 Site 653 (Tyrrhenian sea-western Mediterranean). Memoria, Societá Geologica de Italia, 44, 135-144.

Summerhayes, C.P., Prell, W.L. and Emeis, K.C. 1992. Upwelling Systems: Evolution Since the Early Miocene. The Geological Society of London, Geological Society Special Publication No. 64, 519 pp.

Swan, A.R.H. and Sandilands, M. 1995. Introduction to Geological Data Analysis. Blackwell Science Ltd, Oxford, 446 pp.

Thiede, J., Winkler, A., Wolf-Welling, T., Eldholm, O., Myrhe, A.M., Baumann, K.H., Henrich, R. and Stein, R. 1998. Late Cenozoic history of the polar North Atlantic: Results from ocean drilling. Quaternary Scicence Review, 17, 185-208.

Van Morkhoven, F.P.C.M., Berggren, W.A. and Edwards, A.S. 1986. Cenozoic cosmopolitan deep-water benthic foraminifera. Bulletin des Centres de Recherches Exploration-Production Elf-Aquitaine, Mem. 11, 421 pp.

Yanko, V., Arnold, A.J. and Parker, W.C. 1999. Effects of marine pollution on benthic Foraminifera. In: Modern Foraminifera (Ed. B.K Sen Gupta.). Kluwer Academic Publishers, 217-235.

Zubakov, V. 1992. Correlation of the late Neogene climate events of Atlantic, Mediterranean and Ponto-Caspian. First Congress R.C.A.N.S., Lisboa, 119-122. 